\title{
Avaliação dos prognósticos de precipitação simulada pelo modelo BRAMS na Amazônia Ocidental na estação chuvosa
}

\author{
Janice Freitas LEIVAS ${ }^{1}$, Gustavo Guterres RIBEIRO², Ivan SARAIVA², Julio Silva do Espirito SANTO², \\ Monique Brasil de SOUZA², João Rocha FILHO²
}

\section{RESUMO}

O objetivo deste estudo é avaliar o modelo de previsão numérica do tempo BRAMS (Brazilian Regional Atmospheric Modelling System), a partir da comparação entre os valores preditos e os observados (dados do NCEP/NOAA (National Centers of Environmental Predictions/ National Oceanic and Atmospheric Administration) e do satélite TRMM (Tropical Rainfall Measuring Mission)). O modelo foi assimilado com dados do modelo global do NCEP/NOAA e do CPTEC/INPE. Foram realizadas comparaçóes entre os valores preditos e os observados através da raiz do erro quadrático médio (RMSE) e do erro médio (ME) para os prognósticos de precipitação para os horizontes de 24, 48, 72 e 96 horas, do período de novembro de 2008 a março de 2009. Os resultados mostraram que o modelo BRAMS teve uma performance melhor quando assimilado com dados do modelo global do NCEP/NOAA comparado com as saídas a partir das assimilaçóes do modelo global do CPTEC/INPE.

PALAVRAS-CHAVE: precipitação, modelagem numérica, Amazônia

\section{Evaluation of prognostic of rainfall model BRAMS in Western Amazonia during the wet season}

\section{ABSTRACT}

The objective of this study is to evaluate the model of numerical forecast BRAMS (Brazilian Regional Atmospheric Modelling System) from the comparison between the forecast and observed rainfall (data of NCEP/NOAA (National Centers of Environmental Predictions/ National Oceanic and Atmospheric Administration) and of TRMM satellite (Tropical Rainfall Measuring Mission)). The model used the initial conditions of global model of NCEP/NOAA e do CPTEC/INPE. Comparisons between predicted values and observed through were the root medium square error (RMSE) and of medium error (ME) for the forecast of rainfall of 24, 48, 72 and 96 hours, of the period November of 2008 to March of 2009. The results showed that the BRAMS model had better performance when treated with global model data from NCEP / NOAA compared with the outputs from the assimilation of the global model of CPTEC / INPE.

KEYWORDS: rainfall, numerical modeling, Amazônia

\footnotetext{
1 Embrapa Monitoramento por Satélite (EMBRAPA). Telefone: 19 32116200, fax: 19 32116222. Caixa Postal - 69.049-010 - Manaus - AM, Brasil E-mail: janice@cnpm.embrapa.br

2 Sistema de Proteção da Amazônia - SIPAM. Telefone: 92 33036227, fax: 92 33036289, Caixa Postal - 69.049-010 - Manaus - AM, Brasil.

E-mail: meteorologista@pop.com.br, isaraiva@gmail.com,offsfx@gmail.com, monique-brasil@hotmail.com, rochafi@terra.com.br
} 


\section{INTRODUÇÃO}

O modelo numérico de mesoescala de previsão de tempo BRAMS vem sendo utilizado operacionalmente no SIPAM desde 2006, com a finalidade de atender as exigências advindas de importantes demandas como Forças Armadas, Defesa Civil e fornecer as previsóes do tempo duas vezes ao dia, aos meios de comunicação, escrito, de áudio e televisivos (Dallarosa et al. 2005). As saídas do modelo servem de ferramentas para auxiliar na previsão de tempo para a região Amazônica. São gerados prognósticos de variáveis como temperatura, vento, umidade e precipitação, que se estendem até 96 horas, com intervalo de 6 horas. Porém, a variável meteorológica precipitação é a que desperta maior interesse devido a sua importância para o monitoramento das condiçóes do tempo; de eventos severos; atividades agrícolas, humanas, turismo, entre outras. Importante salientar que a precipitação na Amazônia resulta de sistemas dinâmicos que afetam sazonalmente, sendo mais intensa no verão e outono no hemisfério sul (HS). A precipitação neste período apresenta um padrão característico da circulaçáo, principalmente durante o verão da América do Sul, (Satyamurty et al. 1998). É importante salientar a complexidade da climatologia da precipitação na Amazônia Ocidental devido a vários sistemas meteorológicos atuantes na região. A Amazônia apresenta grande variabilidade da precipitação no tempo e espaço, que está associada a diferentes sistemas de mesoescala, escala sinótica e de grande escala.

O BRAMS, descrito por Cotton et al. (2003), é uma adaptação às condiçóes tropicais do modelo RAMS (Regional Atmospheric Modeling System) (Pielke et al. 1992) desenvolvido por pesquisadores da Universidade do Colorado, Estados Unidos. O modelo resolve as equaçóes da dinâmica de convecção e possui uma série de submodelos que representam processos como a interação solo-vegetação-atmosfera, troca de fluxos turbulentos, transferências radiativas, microfísica de nuvens, entre outros.

No SIPAM, o modelo é inicializado diariamente, sendo obtidas previsóes das condiçóes atmosféricas para 1, 2, 3 e 4 dias, ou seja, horizontes de 24, 48, 72 e 96 horas. As inicializaçôes do BRAMS são realizadas a partir das análises do modelo global T254L64 do NCEP/NOAA (National Centers of Environmental Predictions/ National Oceanic and Atmospheric Administration) e T213L42 do CPTEC/INPE (Centro de Previsão de Tempo e Clima/Instituto Nacional de Pesquisas Espaciais). Para a execução do BRAMS utilizam-se como condiçôes iniciais e de contorno, baixados diariamente via FTP. O modelo também assimila dados iniciais de umidade do solo e dados de temperatura da superfície do mar (TSM) média semanal, topografia e vegetação.

$\mathrm{Na}$ atividade operacional de previsão do tempo, observase que os prognósticos apresentam incertezas, portanto é necessário realizar avaliaçóes dos mesmos, através de comparaçóes com os dados observados em superfície, o que chamamos de "verdade terrestre". Porém, há baixa densidade de dados meteorológicos observados sobre a Amazônia além de serem irregularmente espaçados, fazendo com que a interpolação da precipitação para grade regular apresente uma baixa qualidade, acarretando comprometimento das avaliaçóes do modelo já que a precipitação interpolada não é representativa da realidade. Diante disso, as saídas do BRAMS foram comparadas não somente com dados do NCEP/ NOAA, mas também com as estimativas de precipitação do satélite TRMM (Tropical Rainfall Measuring Mission), já que na região amazônica não são observados erros sistemáticos de sub/superestimativa como no nordeste brasileiro e sul da América do Sul. A utilização dos produtos de estimativa de precipitação, proveniente do satélite TRMM, vem sendo amplamente utilizada para avaliar resultados de modelos numéricos, assim como identificação de pluviômetros com problemas de leitura ou mal localizados (Rozante e Cavalcanti 2008; Collishchonn 2006).

O objetivo deste trabalho é de verificar as previsóes do modelo regional BRAMS através da identificação de falhas espaciais e temporais dos prognósticos obtidos pela diferença entre a previsão e a observação, através de índices estatísticos como raiz do erro quadrático médio (RMSE) e do erro médio (ME). Os dados utilizados para verificação são provenientes do NCEP/NOAA, assim como as estimativas do TRMM, interpoladas para a grade do BRAMS.

\section{MATERIAL E MÉTODOS}

A regiāo de estudo deste trabalho é a Amazônia Ocidental (Figura 1), situada entre as latitudes $5^{\circ} \mathrm{S}$ a $2^{\circ} \mathrm{N}$ e as longitudes $64^{\circ} \mathrm{W}$ a $60^{\circ} \mathrm{W}$, abrangendo os estados Amazonas, Acre, Rondônia e Roraima. O período analisado foi de novembro de 2008 a março de 2009, cujo período compreende a estaçáo chuvosa para a maior parte da regiāo, conforme estudos da climatologia da Amazônia (Molion 1987); Figueroa e Nobre (1990), Fisch (1990), Marengo (1995), Fisch et al. (2007).

A avaliação do modelo numérico de previsão do tempo foi realizada a partir da comparaçáo entre dados observados/ estimados, oriundos do NCEP/NOAA e do TRMM, e os valores preditos pelo BRAMS. Foram analisados os prognósticos de precipitação diária gerados pelo modelo BRAMS, para os horizontes de 24, 48, 72 e 96 horas, através do software GrADS (Grid Analysis and Display System). O BRAMS foi assimilado a partir dos dados do modelo GFS (NCEP/NOAA) e do GAMRAMS (CPTEC/INPE), ambos inicializados às 00UTC. As simulações do BRAMS são realizadas a partir de condiçóes iniciais prescritas e TSM média semanal observada. As condiçôes de contorno usadas para as simulaçóes foram dados de análises do T254L64 e do T213L42, chamados de GFS e GAMRAMS, respectivamente. 


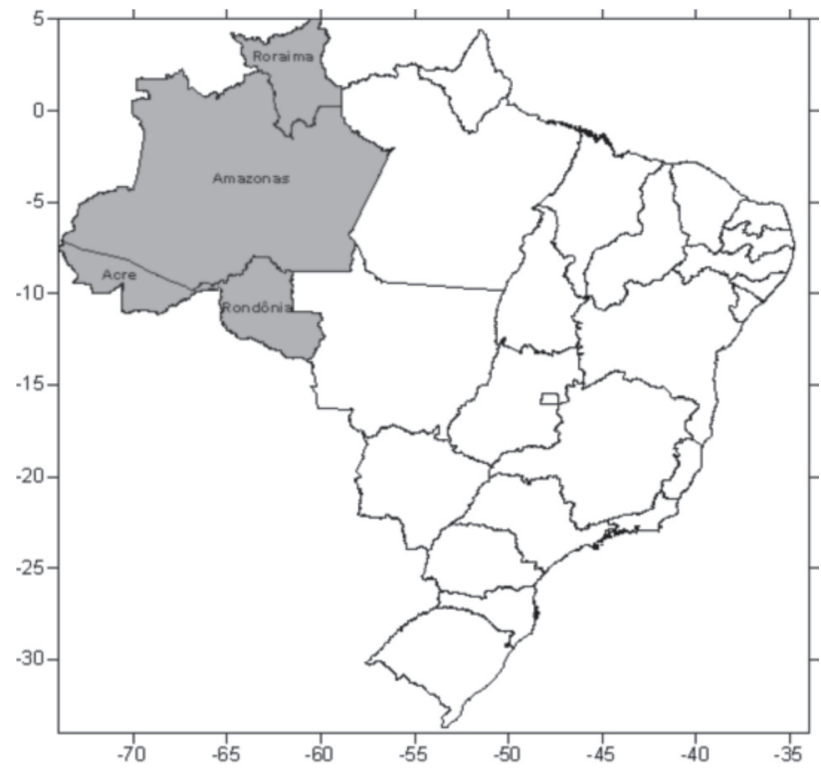

Figura 1 - Localização da área de estudo. Fonte: adaptado de IBGE

Os resultados modelados pelo BRAMS, apresentados em totais de precipitação acumulada a cada 6 horas, foram totalizados para totais diários de precipitaçáo em cada ponto de grade. Procedimento análogo foi realizado com os dados do TRMM (produto 3B42_V6) já que os dados são de estimativas de precipitaçáo de 3 em 3 horas. Devido ao fato de que os dados observados e os prognosticados têm resoluçáo espacial diferente, foi necessário realizar a degradação do modelo e do TRMM para a mesma resoluçáo dos dados do NCEP para fins de comparação já que possui resolução de $1^{\circ}(\sim 111 \mathrm{~km}$ na região tropical), o TRMM 0,25 e o BRAMS com $22 \mathrm{~km}$, com a finalidade de obter resoluçóes iguais e compatíveis. Para isto utilizou-se uma rotina do pacote GrADS.

Para visualizar e comparar os resultados do modelo BRAMS com os dados observados foi realizada uma sobreposição dos resultados modelados pelo BRAMS (GFS e GAMRAMS) e observados (NCEP e TRMM). Primeiro, as imagens com os recortes da grade do modelo foram convertidas em formato texto com suas respectivas coordenadas locais (longitude e latitude) e intensidade de cada pixel, com variação horizontal de $1^{\circ}$.

A partir dos dados de saída do BRAMS, foi realizada uma análise comparativa diária entre os valores simulados e observados de precipitaçáo, para cada ponto de grade. Os dados pluviométricos do TRMM, disponíveis numa grade de $0,25^{\circ}$ (lat/long), foram interpolados para a grade de $1^{\circ}$ de resolução espacial para fins de intercomparaçôes com os dados do modelo.

Para analisar a acurácia dos prognósticos do modelo BRAMS em relação aos dados do NCEP e às estimativas do
TRMM, foram calculados os erros de precipitação prevista em relaçáo à observada, através da raiz do erro quadrático médio (RMSE) e do erro médio (ME), por serem consideradas medidas padrão de precisão das previsôes, capazes de indicar a magnitude do erro. O RMSE dá a medida do erro médio que está sendo cometido. O ME é uma variável que indica a direçáo média dos desvios de um conjunto de previsóes a partir de um conjunto de valores observados. Este valor indica a tendência, se o modelo está superestimando (ME positivo) ou subestimando (ME negativo) (Wilks 1995). As análises estatísticas foram obtidas para as inicializaçôes do BRAMS, a partir dos dados do GFS e do GAMRAMS, para as previsōes de um a quatro dias, conforme esboçado na Figura 2.

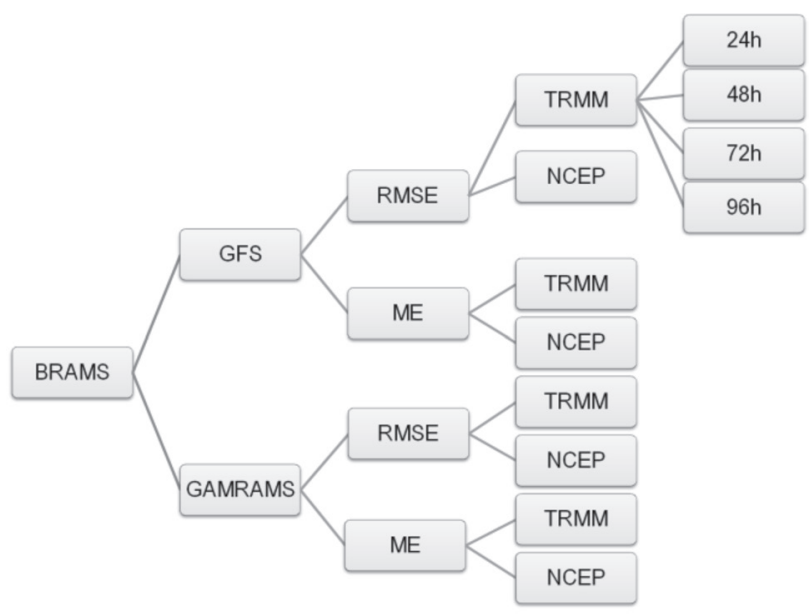

Figura 2 - Fluxograma de execução das análises estatísticas para as inicializações do modelo BRAMS a partir dos dados do GFS e do GAMRAMS e comparações com dados do NCEP e do TRMM, para os horizontes de 24 a 96 horas.

Para verificar a distribuição espacial dos resultados foi utilizado o software SURFER 8.0.

\section{RESULTADOS E DISCUSSÃO}

Com a finalidade de mostrar a diferença entre os dados observados e estimados na Figura 3, é apresentada a precipitação acumulada observada pelo NCEP (Figura 3a) e a estimada pelo TRMM (Figura 3b), para o período de novembro de 2008 a março de 2009. Observa-se que os dados do NCEP foram superiores aos estimados pelo TRMM. Esta diferença entre os dados observados/estimados era esperada, pois conforme foi constatado por Fisch et al. (2007) existe baixa correlação entre dados pluviométricos separados por mais de $5 \mathrm{~km}$ de distância, devido às características de precipitaçáo convectiva, com chuvas localizadas e de curta duraçáo, que ocorre na Amazônia. Vale ressaltar que no período analisado foram registrados altos índices pluviométricos, com anomalias 


\section{PRECIPITACAO ACUMULADA}

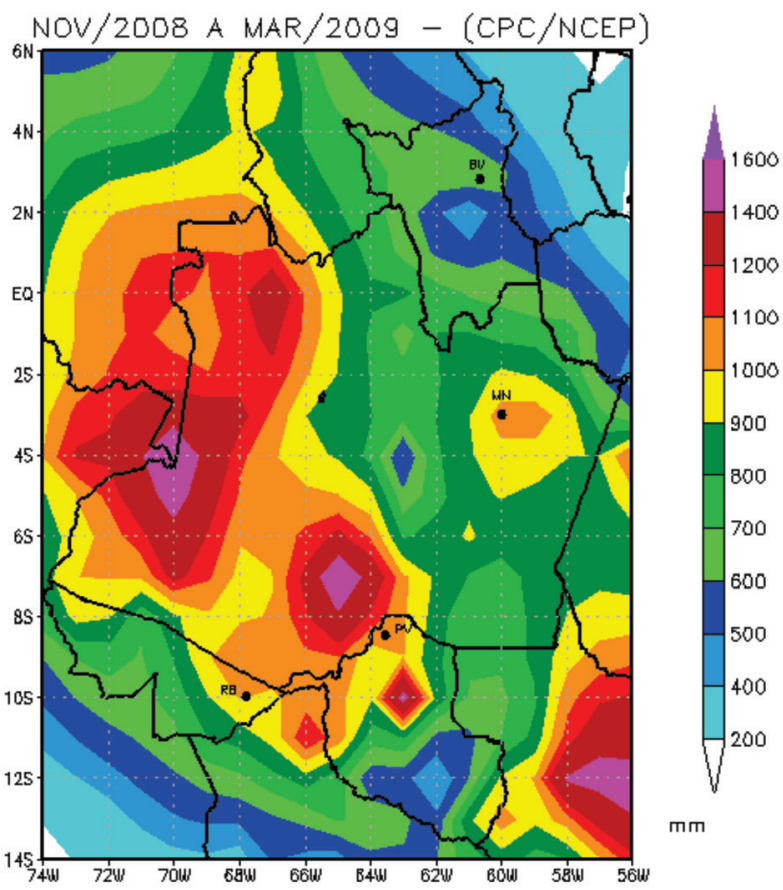

PRECIPITACAO ACUMULADA

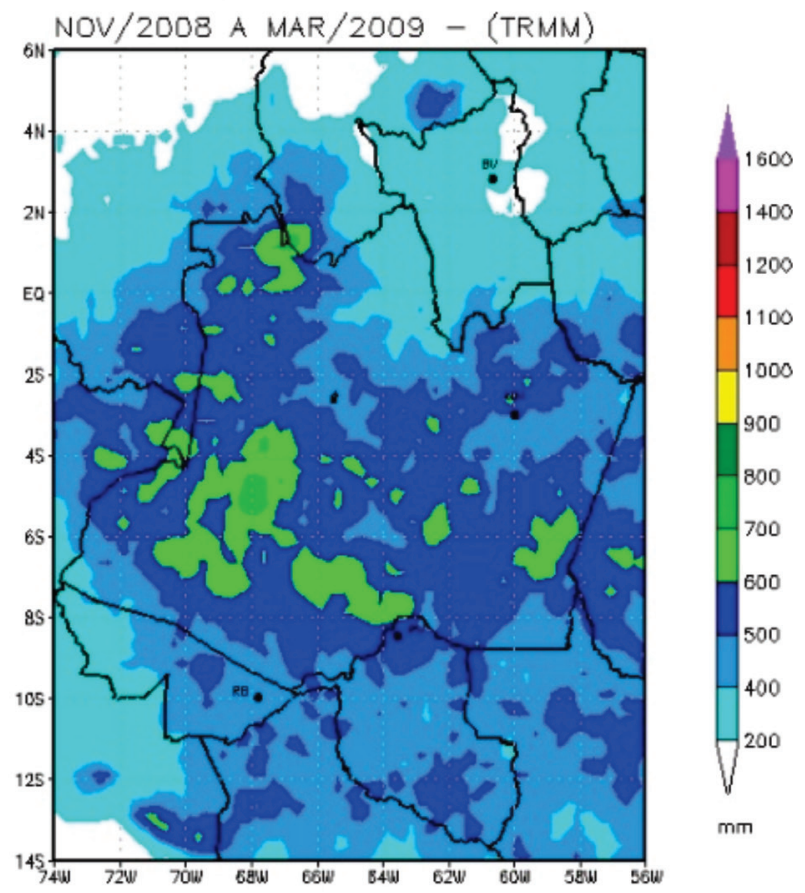

Figura 3 - Precipitação acumulada observada pelo NCEP e a estimada pelo TRMM, dia 26 de dezembro de 2008 na Amazônia Ocidental.

positivas de precipitação no mês de novembro no sudoeste e nordeste do Amazonas e Roraima; e anomalia negativa em Rondônia e Acre. Em dezembro e janeiro, ocorreu anomalia positiva de precipitação em Roraima e no Amazonas. Já em fevereiro, o posicionamento da Zona de Convergência Intertropical (ZCIT) em $4^{\circ} \mathrm{S}$, fez com que a precipitaçáo alcançasse valores superiores ao dobro da normal climatológica no estado do Amazonas. No mês de março, Acre e Amazonas registraram anomalias positivas de precipitação.

A seguir, são apresentadas as análises estatísticas RMSE e EM entre as previsões do modelo BRAMS para a estação chuvosa (novembro de 2008 a março de 2009). As avaliaçóes do modelo foram realizadas a partir dos dados do NCEP e do TRMM, para as inicializações com dados do GFS (Figura 4) e com dados do GAMRAMS (Figura 5), para o conjunto de séries temporais para os horizontes de previsão de 24, 48, 72 e 96 horas. Foi realizada uma análise conjunta entre o RMSE e EM já que o RMSE enfatiza onde ocorrem as maiores diferenças entre o prognosticado e o observado, e o EM posiciona as tendências de sub/superestimativa do modelo.

Na distribuição espacial dos conjuntos de séries temporais para os horizontes de previsão de 24, 48, 72 e 96 horas, para o BRAMS inicializado com dados do GFS, observouse que houve predominância de superestimativa na maior parte da Amazônia Ocidental, com exceção para o norte de
Roraima e sudoeste do Amazonas, onde foram observadas subestimativas de $10 \mathrm{~mm}$. Para o restante da região, observou-se superestimativa de precipitação de 10 a $20 \mathrm{~mm}$. Já no noroeste do Amazonas, os erros alcançaram $50 \mathrm{~mm}$. Para os prognósticos de $48 \mathrm{~h}$ a $96 \mathrm{~h}$, o modelo subestima a precipitação no oeste do Amazonas, em Roraima e no restante da Amazônia, observa-se superestimativa entre 10 e $20 \mathrm{~mm}$. Foram observados erros elevados em todas as análises (RMSE) na regiáo noroeste do estado do Amazonas. Isto deve ter ocorrido devido ao efeito da topografia. Uma possível causa de erros elevados das previsóes do BRAMS para a regiáo com relevo acentuado pode ser a náo representaçáo da topografia local através da resolução horizontal, utilizada nas simulaçôes $(20 \mathrm{~km})$, acarretando impacto significativo nas variáveis de superfície. Quando o modelo BRAMS foi inicializado com dados do GAMRAMS e comparado com dados do NCEP (Figura 5a), observou-se que com o aumento dos horizontes de previsão de um a quatro dias, os erros foram aumentando alcançando $40 \mathrm{~mm}$, em média, na região sudoeste da Amazônia Ocidental. Houve predomínio de superestimativa na Amazônia Ocidental, com exceção ao norte de Roraima, para prognósticos de 24 horas, e para o centro do Amazonas, para $48 \mathrm{~h}$, onde foram observadas subestimativas. Na regiáo com elevada topografia (noroeste do Amazonas) persistiram os elevados erros, já observados quando o modelo foi inicializado com dados do GFS, em torno de $50 \mathrm{~mm}$. 


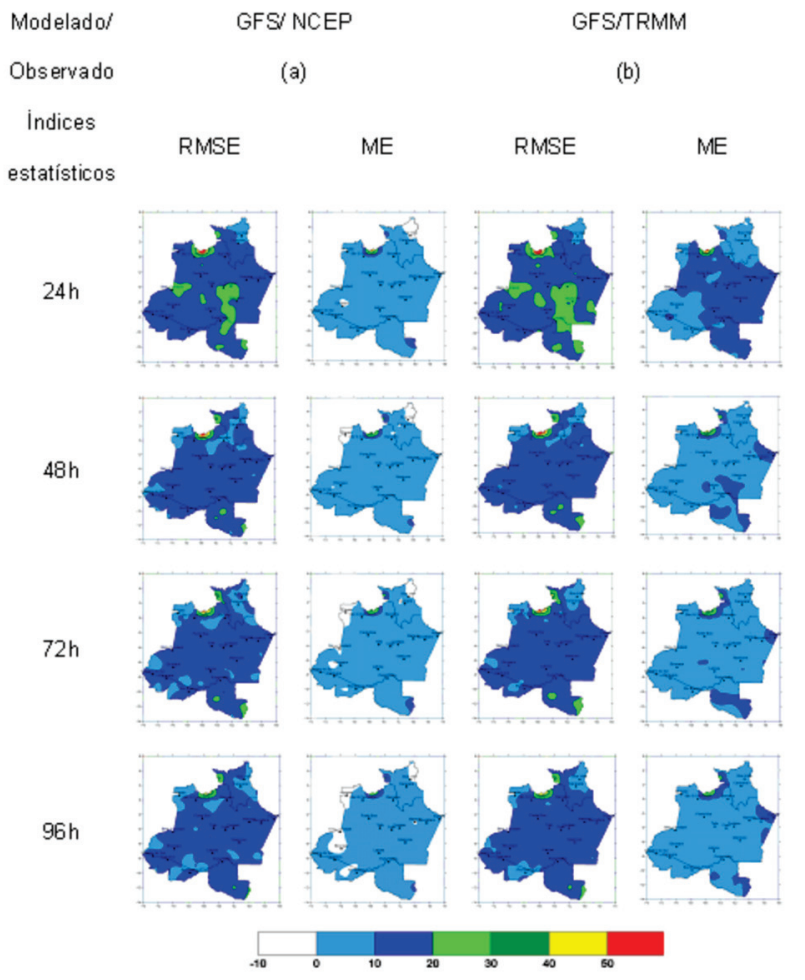

Figura 4 - Distribuição espacial do RMSE e EM do BRAMS inicializado com dados do GFS comparados com NCEP e do TRMM.

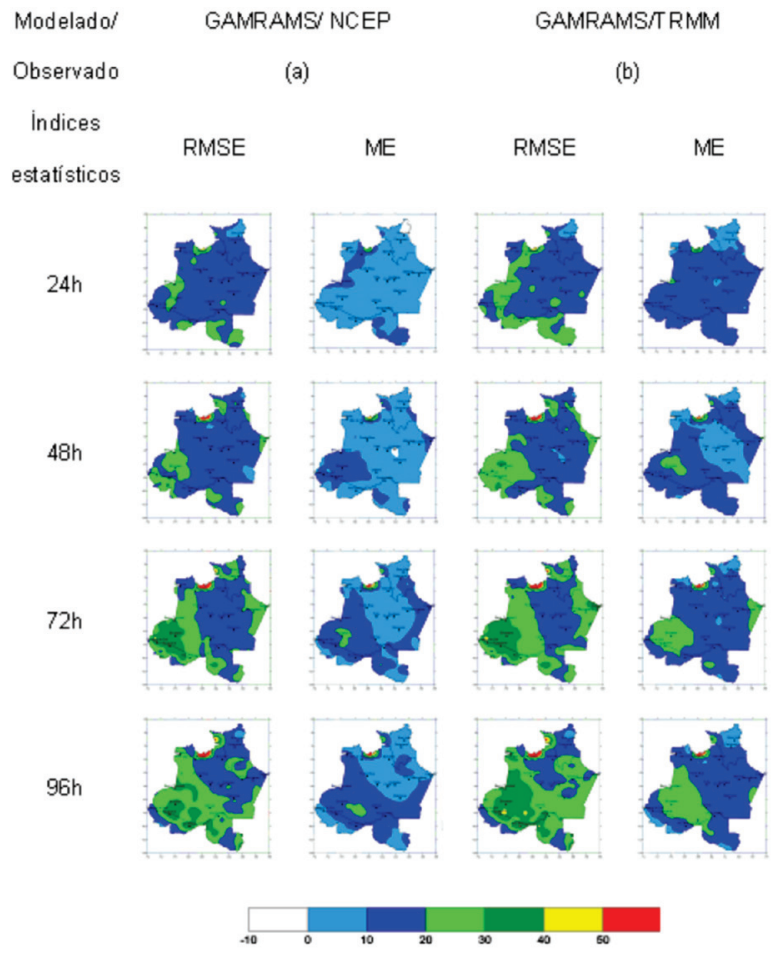

Figura 5 - Distribuição espacial do RMSE e EM do BRAMS inicializado com dados do GAMRAMS comparados com NCEP e do TRMM
Comparando as saídas do BRAMS com as estimativas do TRMM, observou-se que as saídas do modelo inicializadas a partir dos dados do GFS (Figura 4b) foram mais precisas do que quando inicializadas com o GAMRAMS (Figura 5b). Em todos os prognósticos, foi constatada superestimativa, principalmente, nas primeiras 24 horas. Os núcleos de elevados erros na regiáo onde se localiza o Pico da Neblina, no norte do Amazonas, persistiram em todos os prognósticos. Houve predomínio de erros entre 10 e 20 mm, com exceção de áreas no norte de Roraima e no noroeste do Amazonas. Com o avanço dos horizontes de previsão, as superestimativas do modelo ficaram mais evidentes.

Com a inicialização a partir do GAMRAMS e comparada com NCEP (Figura 5a) observou-se que com o aumento dos horários de previsão, os erros foram sendo acumulados, ou seja, a previsão para 24 horas foi mais precisa que a de 48 , 72 e 96 horas, sucessivamente, predominando RMSE de 20 a $40 \mathrm{~mm}$. O núcleo de elevados erros na região com elevado relevo, no noroeste do Amazonas foi intensificado.

$\mathrm{Na}$ distribuiçáo espacial dos erros do modelo a partir da inicialização com dados do GAMRAMS (Figura 5), foi observado o aumento dos erros à medida que aumenta o horizonte de previsão. $\mathrm{Na}$ Amazônia Ocidental predominavam erros de $10 \mathrm{~mm}$ e com o avanço do horizonte dos prognósticos, foram observadas áreas com erros de $20 \mathrm{~mm}$ na maior parte da região. Os núcleos de elevados erros no noroeste do Amazonas foram observados em todos prognósticos, de 24 a 96 horas. Era esperado que com o avanço dos horizontes de previsão, os erros fossem aumentando já que as previsôes seguintes ocorrem a partir do prognóstico de 24 horas, ou seja, previsão a partir de prognósticos, acarretando acúmulo de erro.

$\mathrm{O}$ modelo superestima a precipitação em praticamente toda a região, o que pode ser devido ao fato de que o mesmo não consegue detectar chuvas isoladas na região, como as oriundas de sistemas convectivos. É observado um erro sistemático na parte noroeste do estado do Amazonas.

Os resultados encontrados corroboram com os estudos de Rogers et al. (1996) que observaram que o ETA apresenta grande variação da precipitação devido à influência da topografia. Machado et al. (2002) observou que os sistemas convectivos de mesoescala formam-se, preferencialmente, sobre regiôes mais elevadas indicando que a topografia é um fator dominante mesmo na Amazônia. Os resultados encontrados corroboram com os obtidos por Alves et al. (2000) que observaram superestimativa no modelo ETA, nos estados do Amazonas e Roraima. Os resultados obtidos evidenciam que os prognósticos apresentam incertezas que podem ser devido às condiçóes iniciais ou ao efeito da orografia, como a náo representação de processos físicos causados pelo relevo, corroborando com Bustamante et al. (1999); Chou e Justi da Silva (1999). 
A acurácia dos prognósticos obtidos pelos modelos regionais de previsão numérica de tempo depende das condiçôes iniciais para inicializar o modelo, da resoluçáo espacial e das parametrizaçóes físicas do modelo. Zorita e Storch (1999) ressaltam que as falhas dos prognósticos dos modelos podem ser devido à resoluçáo espacial incompatível com as características da superfície terrestre e aos processos da escala de subgrade, tais como formação de nuvens, chuva, infiltração, evaporação, que não são parametrizados adequadamente.

Analisando-se as previsões diárias do BRAMS, observou-se que em alguns eventos o modelo produz previsóes de baixa qualidade, podendo posicionar o sistema erroneamente, subestimando ou superestimando a sua intensidade, na maior parte da regiáo, enquanto que em outros eventos o modelo indica corretamente a ocorrência. Uma possibilidade da fonte de erros do modelo pode ser devido às saídas do modelo representarem um valor médio da grade, enquanto que a variável precipitação apresenta grande variação espacial. Olson et al. (1995) salientaram que a complexidade física dos processos de precipitaçáo e as escalas de tempoespaço, envolvidas em tais processos, não são resolvidas satisfatoriamente pelos modelos numéricos. Mesmo os modelos com alta resoluçáo de Previsão Numérica de Tempo (PNT) náo conseguem estimar satisfatoriamente os campos de precipitaçáo por meio de suas parametrizaçóes. No período analisado foi observado que ocorreu náo somente um aumento do erro como também a variabilidade do erro deteriorando a previsibilidade de tempo, principalmente, quando o modelo BRAMS foi inicializado com dados do GAMRAMS, apresentando melhores prognósticos quando inicializado com dados do GFS.

\section{CONSIDERAÇÕES FINAIS}

Neste estudo, a partir da comparaçáo entre a variabilidade da precipitação prevista pelo modelo BRAMS para a Amazônia Ocidental e dados observados, ressalta-se que para o período de novembro de 2008 a março de 2009, o modelo BRAMS foi um bom indicador de ocorrência de precipitação, apesar da tendência de superestimativa, principalmente no noroeste do Amazonas. Os prognósticos de precipitação do modelo BRAMS assimilados com os dados do GFS mostraram-se mais adequados do que quando assimilados com dados do GAMRAMS.

Os resultados encontrados podem servir para afirmar a necessidade de aperfeiçoamento dos métodos de previsão para elaborarem prognósticos cada vez melhores para a regiáo estudada. Para trabalhos futuros, pretende-se aumentar o período de verificação, expandindo para a estaçáo seca (de maio a setembro) e períodos de transição como outubro e abril, com a finalidade de conhecer os erros característicos e regióes onde se concentram as maiores discrepâncias entre o previsto e o observado e subseqüente identificação das possíveis parametrizaçoes responsáveis pelos erros, permitindo melhorias nas previsóes de tempo.

\section{AGRADECIMENTOS}

Os autores agradecem ao SIPAM que financia o desenvolvimento desta pesquisa, com o apoio da FAPEAM (Fundação de Amparo à Pesquisa no Estado do Amazonas).

\section{BIBLIOGRAFIA CITADA}

Alves, L.M.; Melo, A.B.C.M.; Chou, S.C; Nobre, P. 2000. Estudo comparativo entre a precipitaçáo sazonal simulada pelo modelo ETA e observada sobre o Brasil, p. 1029-1035. In: Congresso Brasileiro de Meteorologia, XI.

Bustamante, J.F.; Gomes, J.L.; Chou, S.C.; Rozante, J.R. 1999. Evaluation of april 1999 rainfall forecasts over South America using the Eta Model. Climanalise, 14(5): 1-17.

Chou, S.C.; Justi, M.G.A. 1999. Objective evaluation of ETA model precipitation forecasts over South America. Climanalise, 1: 1-17.

Collishchonn, B. 2006. Uso de precipitação estimada pelo satélite TRMM em modelo hidrológico distribuido. Dissertação de mestrado, Instituto de Pesquisas Hidrológicas/Universidade Federal do Rio Grande do Sul, Porto Alegre, Rio Grande do Sul. 193 pp.

Cotton, W.R.; Pielke, R.A.; Walko, R.L.; Liston, G.E.; Tremback, C.J.; Jiang, H.; Mcanelly, R.L.; Harrington, J.Y.; Nicholls, M.E.; Carrio, G.G.; Mcfadden, J.P. 2003. RAMS 2001: Current status and future directions. Meteorology and Atmospheric Physics, 82: 5-29.

Dallarosa, R.G.; Senna, R.C.; Saraiva, J.M.B. 2005. O Projeto SIVAM, o SIPAM e sua contribuiçáo para a segurança da navegação aérea na Amazônia Legal. Boletim da Sociedade Brasileira de Meteorologia, 29(3): 27-30.

Figueroa, S.N; Nobre, C.A. 1990. Precipitations distribution over central and western tropical South America. Climanalise, 5(6): 36-45.

Fisch, G.; Vendrame, I. F.; Hanaoka, P.C.M. 2007. Variabilidade espacial da chuva durante o experimento LBA/TRMM 1999 na Amazônia. Acta Amazonica, 37: 583-590.

Fisch, G.1990. Climatic Aspects of Amazonian Tropical Forest. Acta Amazonica, 20: 39-48.

Machado, L.A.T.; Laurent, H.; Lima, A.A. 2002. Diurnal marcho $\mathrm{f}$ the convection observed during TRMM-WETAMC/LBA. Journal Geophysical Research, 107: 31.1-31-15.

Marengo, J. 1995. Interannual variability of deep convection in the tropical South American sector as deduced from ISCCP C2 data. International Journal of Climatology, 15(9): 995-1010.

Molion, L.C.B. 1987. Climatologia dinâmica da região amazônica: mecanismos de precipitação. Revista Brasileira de Meteorologia, 2: $107-117$. 
Olson, D.A.; Junker, N.; Korty, B. 1995. Evaluation of 33 years of quantitative precipitation forecasting at the NMC. Weather and Forecasting, 10(3): 498-511.

Pielke, R.E.; Cotton, W.R.; Walko, R.L.; Tremback, C.J.; Lyons, W.A., Grasso, L.D.; NicholIs, M.E., Moran, M.D.; Wesley, D.A., Lee, T.J.; Copeland, J.H. 1992: A Comprehensive Meteorological Modeling System - RAMS. Meteorology and Atmospheric Physics, 49: 69-91.

Rogers, E.; Black, T.L.; Deaven, D.G.; DiMego, G.J, Zhoo, Q.; Baldiwm, M.; Junker, N.W.; Lin, Y. 1996. Changes to the operacional "early" ETA analysis/forecast system. Weather Forescasting, 11: 391-413.

Rozante, J.R. Cavalcanti, I.F.A, 2008. Regional ETA experiments: SALLJEX and MCS development. Journal of Geophysical Research, 113.
Satyamurty, P.; Nobre, C.A.; Dias, P.L.D. 1998. Tropics - South America Southern Hemisphere Meteorology. American Meteorological Society, 27: 201- 241.

Wilks, D.S. 1995. Forecast verification, p. 255-336. In: Statistical methods in the atmospheric sciences. v.1. Cornell University, San Diego, United States of America.

Zorita, E.; Storch, H.V. 1999. The analog method as a simple statistical downscaling techinique: comparison with more complicated methods. Journal of Climate, 12: 2474-2488.

Recebido em 26/04/2010

Aceito em 14/02/2011 